\title{
Qualissus-redes e as proposições do banco mundial: problematizações críticas
}

\author{
Qualissus-network and the proposals of the world bank: criticals problematizations
}

Qualissus-red y las proposiciones del banco mundial: problematizaciones críticas

Recebido: 10/05/2015

Aprovado: 20/02/2016

Publicado: 01/05/2016

\author{
Marina Monteiro de Castro e Castro ${ }^{1}$
}

O presente artigo tem por objetivo apresentar problematizações críticas acerca do QualiSUSRedes - Programa do Ministério da Saúde financiado pelo Banco Mundial. Através de uma pesquisa junto aos documentos que subsidiam o programa e às referências teóricas que tratam do mesmo, foi realizada uma apreciação das principais propostas do Programa. 0 estudo demonstrou que, apesar do QualiSUS-Redes ter em sua composição direcionamentos que buscam avanços na qualificação dos serviços públicos de saúde, é necessário problematizar o alinhamento aos ditames do Banco Mundial, em um contexto de ataque às políticas públicas de saúde, para desmistificar os fundamentos políticos e econômicos dos programas governamentais.

Descritores: Banco Internacional para Reconstrução e Desenvolvimento; Política de saúde; Saúde pública.

This article aims to present criticals problematizations about QualiSUS-networks - Ministry of Health of Brazil, program funded by the World Bank. Through a study conducted by the documents that support the program and the theoretical references that deal with the same, an appreciation of the main proposals of the program was held. The study showed that despite the QualiSUS-nets have in their composition directions seeking advances in terms of public health services, it is necessary to question the alignment to the World Bank dictates in an attack context of public health policies, to demystify political and economic foundations of government programs.

Descriptors: International Bank for Reconstruction and Development; Health policy; Public health.

Este artículo tiene como objetivo presentar problematizaciones críticas sobre el QualiSUSredes - del Ministerio de Salud del Brasil, programa financiado por el Banco Mundial. Através de una investigación hecha por los documentos que apoyan el programa y las referencias teóricas que tienen que ver con el mismo, se hice una apreciación de las principales propuestas del programa. El estudio mostró que a pesar del QualiSUS-redes tener en sus direcciones de composición la busqueda de avances en los servicios de salud pública, es necesario cuestionar la líneas seguidas a los ditames del Banco Mundial en un contexto de ataque de las políticas de salud pública, para desmitificar fundaciones políticas y económicas de los programas de gobierno.

Descriptores: Banco Internacional de Reconstrucción y Fomento; Política de salud; Salud pública.

\footnotetext{
${ }^{1}$ Assistente Social. Especialista em Docência em Saúde. Especialista em Políticas e Pesquisa em Saúde Coletiva. Mestre e Doutora em Serviço Social. Professora Adjunta da Faculdade de Serviço Social da Universidade Federal de Juiz de Fora/MG. marinamccastro@yahoo.com.br. Brasil.
} 


\section{INTRODUÇÃO}

O QualiSUS - Política de qualificação da atenção à saúde no Sistema Único de Saúde (SUS), foi lançado em 2003 com o discurso da necessidade de elevar a qualidade da assistência prestada na área da saúde, tendo como pilar a humanização. A política foi dinamizada com o "Programa de Formação e Melhoria da Qualidade das Redes de Atenção à Saúde (RAS) - QualiSUS Redes" apresentado em 2009, que propõem apoio à organização das redes regionalizadas de atenção à saúde no Brasil. É um projeto de cooperação entre o Banco Mundial e o Ministério da Saúde.

0 programa segue a lógica do Banco Mundial de financiamentos regionalizados e, propõem o desenvolvimento de uma ação que incorpore toda a rede de saúde e tenha como foco as Doenças Crônicas não Transmissíveis (DCNTs), uma vez que "o fortalecimento do capital humano é essencial para a agenda do crescimento, em termos do desenvolvimento de uma força de trabalho mais qualificada, saudável e ágil, capaz de inovar e de se adaptar às novas tecnologias para aumentar a produtividade"1.

No QualiSUS, a Política Nacional de Humanização (PNH) é destacada como base para seu desenvolvimento, sendo elencados a necessidade de valorização dos trabalhadores, mudanças nas inter-relações entre profissionais e usuários e os novos modelos de gestão.

Em busca da chamada "qualidade dos serviços de saúde" e do reforço à humanização da saúde, o QualiSUS demonstra a vinculação da política com as necessidades de fomento da economia, ao ser destacado a necessidade de melhoria da capacidade de gestão, controle social, superação de iniquidades, integralidade e racionalidade dos investimentos em infraestrutura, a partir do reforço da articulação entre a lógica econômica e sanitária.

Neste sentido, o presente artigo tem como objetivo apresentar problematizações críticas acerca do QualiSUs-Redes - programa do Ministério da Saúde financiado pelo Banco Mundial.

\section{MÉTODO}

Esta é uma reflexão com o uso da pesquisa bibliográfica que se utilizou de fontes primárias e secundárias. Foram levantados documentos do Ministério da Saúde sobre o QualiSUS-Redes, humanização e qualidade em saúde, e documentos do Banco Mundial direcionados ao Brasil, que subsidiam o Projeto QualiSUS-Redes.

Utilizaram-se também fontes secundárias, isto é, informações que já foram trabalhadas por outros estudiosos e já estão sob o domínio público. Estas fontes foram coletadas, principalmente, na base de dados "Scielo". Foi realizada pesquisa com a palavra-chave "QualiSUS", sendo encontrados três artigos que se referem aos serviços no âmbito hospitalar ou de urgência e emergência, de autoria de Gusmão-Filho et al, 20102, O'Dwyer et al, 20093; Bittencourt e Hortale, $2007^{4}$.

\section{RESULTADOS}

o QualiSUS-Rede e suas diretrizes operacionais foram dispostas na Portaria no396 de 4 de março de 2011. Esta indica que o programa fornece materialidade aos movimentos em torno da consolidação do SUS, representados no Pacto pela Saúde. É definido como uma estratégia de apoio à organização das redes regionalizadas de atenção à saúde no Brasil para qualificar a atenção e gestão em saúde e do debate das temáticas da atenção e qualificação do cuidado em saúde.

Os objetivos do QualiSUS-Rede são assim expressos:

I - a organização, no âmbito do SUS, de redes de atenção à saúde que considerem o protagonismo da atenção primária no seu ordenamento;

II - a priorização dos investimentos na atenção especializada (ambulatorial e hospitalar), na atenção de urgência e emergência e no aprimoramento dos sistemas logísticos de suporte à rede; III - o aumento da eficiência alocativa e produtiva do SUS; 
IV - a instituição e o aprimoramento dos mecanismos de gestão das redes de atenção à saúde;

V - o fortalecimento da regionalização, da contratualização, da regulação do acesso, da responsabilização dos gestores e da participação social;

VI - a qualificação do cuidado em saúde, incentivando a definição e implantação de protocolos clínicos, linhas de cuidado e processos de capacitação profissional;

VII - a melhoria da efetividade e da resolubilidade da prestação dos serviços de saúde para as populações cobertas pelo projeto;

VIII - a produção, a sistematização e a difusão dos conhecimentos voltados à melhoria da qualidade da atenção e da gestão em saúde, ao desenvolvimento de metodologias $\mathrm{e}$ processos de avaliação e gestão da qualidade e à gestão da inovação tecnológica em saúde.

De acordo com CONASS $^{6}$, as proposições para o QualiSUS devem estar afinadas com o novo contexto políticoinstitucional trazido pelo Governo Dilma e com os documentos e legislações recentes, sendo destacado: a Portaria GM no 4279 de 2010 que estabelece diretrizes para a organização da Rede de Atenção à Saúde no âmbito do Sistema Único de Saúde; Programa Nacional de Melhoria do Acesso e Qualidade na Atenção Básica (PMAQ); Decreto Federal nำ7508 de 2011 que regulamenta a Lei no8. 080 de 1990 e o Plano Nacional de Enfrentamento as Doenças Crônicas Não transmissíveis (DCNT).

Para desenvolvimento deste programa, foi firmado um acordo de empréstimo com o Banco Mundial em 2009 (ainda no Governo Lula) que trouxe como mote para a política de saúde o apoio à organização de Redes de Atenção à Saúde no Brasil. Para execução do Projeto, foi firmado também um termo de cooperação em 2010, entre o Ministério da Saúde e a Fundação Oswaldo Cruz (FIOCRUZ).

Durante o governo Lula (2003-2010), tal projeto se desenvolveu pontualmente e mais direcionado para os setores de urgência e emergência. É somente no Governo Dilma (iniciado em 2011) e com a aprovação do financiamento do Banco Mundial, que o QualiSUS-Rede irá avançar, principalmente no âmbito de sua regulamentação com as Portarias no 396 de 2011 e 1.375 de 2012.

No primeiro ano do mandato foi estabelecido como prioridade a organização das Redes de Atenção à Saúde (RAS) em consonância com o QualiSUS-Rede e a transferência de responsabilidade de execução do projeto para o Ministério da Saúde.

$\mathrm{Na}$ Nota Técnica divulgada pelo CONASS (Conselho Nacional de Secretários Estaduais de Saúde) em 19 de abril de 2013 é realizada uma análise do histórico e da situação atual do projeto, que indicou que com a eleição de Dilma, o Ministério da Saúde em conformidade com o Banco Mundial, estabeleceram como iniciativas prioritárias para o QualiSUS-Redes: curso de ambiência em saúde para profissionais de arquitetura e engenharia das secretarias estaduais e municipais de saúde das regiões do projeto QualiSUS-Redes; qualificação da gestão das tecnologias médico-hospitalares nas redes de atenção à saúde; intervenção sistêmica da assistência farmacêutica nas redes de atenção à saúde das regiões do projeto QualiSUS-Redes; implantação da gestão de custos em hospitais e nas redes de atenção à saúde das regiões selecionadas; e estratégia de transporte de pessoas para procedimentos eletivos.

Na avaliação social realizada pelo Banco Mundial para o estabelecimento do contrato do QualiSUS-Rede, o Banco considerava que deveriam ser incluídos no projeto: a mitigação das desigualdades na condição de saúde, a melhoria do acesso e uso de assistência de média e alta complexidade entre a população pobre, abordar as doenças não transmissíveis entre as populações pobres e melhorar a satisfação do paciente em relação à assistência financiada pelo SUS.

Na redefinição dos eixos prioritários do QualiSUS-Rede é possível notar o enfoque dado às diretrizes expressas pelo Banco Mundial, principalmente no que se refere a ênfase na gestão e em estratégias que viabilizem a articulação entre expansão da 
rede de saúde e incentivo à acumulação de capital.

Desta forma, o projeto proposto estaria alinhado aos pilares estratégicos da equidade, governança e competitividade e com a estratégia global da saúde para o Brasil de aumento da qualidade dos cuidados e melhoria dos resultados em saúde e de contribuição para competitividade e crescimento do Brasil, melhorando a eficiência e acessibilidade dos gastos sociais, a produtividade da força de trabalho e fortalecendo a governança através da introdução de mecanismos de responsabilização na área da saúde ${ }^{7}$.

É ressaltado ainda pelo Banco que o QualiSUS-Rede está articulado com o PACMais Saúde e com o Pacto pela saúde (2006) e aponta que esta articulação é um dos ganhos do projeto, pois o PAC trouxe reconfigurações dos sistemas de saúde via "Regional Health Care Networks (RHCN)" ou rede regionalizada de saúde e a introdução de gestão baseada em resultados; e os Pactos pela Saúde regulamentaram a regionalização dos serviços de média e alta complexidade, a definição do papel dos estados e sua responsabilização pelos sistemas de prestação de serviços de saúde. Outro ganho seria a flexibilidade na governança e gestão geradas pela formação dos consórcios intermunicipais.

As interferências do Banco Mundial aparecem claramente, pois nos documentos direcionados ao Qualisus destaca que contribuirá com a experiência que possui nas questões relacionadas ao financiamento da saúde, formato de redes de cuidados, especialmente para doenças não transmissíveis, governança, gestão de recursos e melhoria de desempenho, que seriam as áreas "onde o know-how está faltando no Brasil"8.

Desta forma, pode-se observar que a perspectiva de garantia de financiamento pelo Banco Mundial para o QualiSUS-Rede acompanha as propostas de contrarreforma na política de saúde desenvolvidas desde o final dos anos 1980, e é posto como o elo que faltava no Brasil entre a Pasta da Saúde e o Banco Mundial, no sentido de reforçar a eficiência, qualidade e governança, principalmente no que tange à média e alta complexidade, pois estes não são cobertos por outros contratos com o Banco.

\section{DISCUSSÃO}

A partir do contexto apresentado é possível identificar que os objetivos traçados para o QualiSUS-Redes contemplam elementos importantes para qualificação dos serviços públicos de saúde. No entanto, é perceptível que os mesmos estão centrados na melhoria da eficácia do sistema financeiro do SUS, no trato das doenças não transmissíveis, e melhoria da qualidade e eficiência dos serviços de saúde financiados pelo SUS, com ênfase nos hospitais de nível secundário, especialidade, diagnóstico e centros de urgência e emergência, tendo por base as proposições do Banco Mundial para a política de saúde brasileira.

Tais direcionamentos se contrapõem diretamente a construção de uma saúde pública, com gestão democrática e com o perfil de uma saúde "humanizada" centrada na atenção primária e no acesso da população aos serviços que de fato atinjam as suas necessidades de saúde.

No âmbito dos documentos do Banco Mundial, há a ênfase no apontamento de que, apesar dos avanços gerados com o SUS, os principais problemas do setor saúde no Brasil são a baixa capacidade gerencial e a falta de orientação de desempenho que compromete a capacidade de obter grandes ganhos. A ineficiência e baixa qualidade dos serviços são postas como decorrentes da elevada dívida e carga fiscal pública. E, por isso, os crescentes custos dos cuidados em saúde tem gerado preocupação das autoridades.

Desta forma, o programa tem uma contradição expressa ao atender as demandas e necessidades da classe trabalhadora e, ao mesmo tempo, garantir a maximização da lucratividade do capital. Esta contradição se acentua ao incorporar no âmbito da saúde pública a lógica privada, principalmente no âmbito da gestão, "sob a forma de uma racionalidade empresarial e 
seus instrumentos administrativo-gerenciais, como a contratualização por metas" 9 .

Nota-se que as contradições existentes na política de saúde são delegadas à gestão e não aos problemas concernentes a submissão da política social à econômica e a lógica privilegiada pelo Estado para as políticas públicas. Desta forma, a partir de uma análise liberal, o fracasso do modelo de gestão é responsabilizado pela crise da saúde, sendo ressaltada a necessidade de adoção para a área de uma perspectiva gerencial e de mercado para solucionar os problemas vivenciados.

No documento publicado pelo Governo Lula com o balanço dos seus dois mandatos intitulado "Brasil 2003-2010", é apontado que um novo direcionamento foi estabelecido pela saúde no Brasil a partir de 2003. Interessante observar que neste documento está posto que a saúde foi tratada neste período no contexto das políticas sociais e dos direitos de cidadania, mas também passou a ser considerada central para o projeto de desenvolvimento nacional através da articulação entre as dimensões social, econômica e tecnológica do setor. Desta forma, "reconheceu-se o caráter estratégico do chamado Complexo Industrial da Saúde (CIS), que envolve todas as atividades produtivas em saúde, abarcando tanto $\mathrm{o}$ setor industrial quanto $\mathrm{o}$ de serviços" 10 .

A eleição de Dilma em 2010 foi legatária da trajetória do Governo Lula e do consenso social construído no seu primeiro governo (2011-2014). No seu primeiro mandato e início do segundo, é possível observar que as posições assumidas indicam um governo ainda mais privatista $\mathrm{e}$ comprometido com o atual modelo econômico. Dilma iniciou seu governo com cortes orçamentários, restrição de investimentos e privatizações. Ao mesmo tempo um aspecto importante é a crescente insatisfação dos grupos sociais quanto à condução do governo, como poder se visto nas atuais manifestações ${ }^{11}$.

Neste sentido, é importante ressaltar a importância que o Banco Mundial tem na indução dos processos de "reforma do
Estado" em um contexto de crise do capital. Desde os anos 1990 o Banco Mundial vem ampliando suas estratégias ideológicas de aceitação da agenda neoliberal, indicando a pauta da reforma, quadros para a formulação de políticas, provisão de recursos para aliviar tensões sociais e dividir a oposição política e a intermediação de acordos internacionais "para elevar os custos políticos dos governos que decidissem - ou fossem levados a- trilhar uma rota alternativa de desenvolvimento"12.

É neste contexto que a retórica neoinstitucional, da noção de governança e de reformas, passa a ser pauta central para o Banco Mundial no sentido de fortalecer os Estados nacionais no aceleramento, aprofundamento, e consolidação da reestruturação do capital.

Para os países de capitalismo periférico, como o Brasil, esta pauta não será diferente, influenciando diretamente na elaboração, organização e execução da política de saúde. De uma forma geral, a linha político-institucional adotada pelo QualiSUS Redes traz indicativos de aproximação às prioridades do Banco Mundial e de necessidade de tratar a política em sua relação com o desenvolvimento e a política econômica brasileira.

Pode-se visualizar esse direcionamento por meio dos respaldos utilizados para o desenvolvimento do Projeto. 0 decreto no 7.508 , por exemplo, tem forte ênfase à parceria público-privada e desqualifica os conselhos de saúde como espaço de controle social das políticas de saúde.

No PMAQ, pode ser visto que, para o objetivo de qualificar a atenção ofertada na atenção básica, são privilegiadas alterações nos mecanismos de gestão, ambiência, padrões e indicadores para mensurar as ações desenvolvidas. É proposto um "processo de mudança de cultura de gestão", com gestão dos recursos em função dos compromissos e resultados pactuados e alcançados e o estímulo para que trabalhadores orientem os serviços em função das necessidades e da satisfação dos usuários. 
No que tange aos documentos do Pacto pela Saúde, Plano Mais Saúde e do projeto de Lei sobre as fundações estatais, Soares ${ }^{13}$ indica que estes incorporam a racionalidade da contrarreforma na política de saúde em três eixos centrais que englobam a vinculação da saúde com desenvolvimento, a redefinição do público estatal e a incorporação de "novos" modelos de gestão.

A indicação do Banco Mundial para o trato das doenças não transmissíveis no QualiSUS-Redes se refere ao fato que, hoje, no Brasil, o maior número de mortes é gerada por doenças cardiovasculares e respiratórias e crônicas, o que interfere na produtividade do país.

Para análise, avaliação e desenvolvimento do projeto QualiSUS-Rede é apontado como base o Relatório do Banco Mundial de 2007 - que traz diversos eixos de incorporação de uma lógica privatista na política pública de saúde. Neste relatório é exposto o aumento da mortalidade e acometimento da população brasileira por doenças não transmissíveis, principalmente doenças cardiovasculares, crônicas (hipertensão e diabetes) e câncer, que incidem diretamente na perda de produtividade do trabalhador, e, por isso, há um custo adicional para os serviços de saúde e uma queda na produtividade do trabalhador. " 0 custo adicional de tratamento combinado com a perda da produtividade (devido à morte prematura e invalidez) poderia consumir um adicional de $5 \%$ do Produto Interno Bruto (PIB)"1.

É notório, assim, que os eixos do programa obedecem a uma lógica determinada pelos interesses do capital voltada para a reprodução da força de trabalho, uma vez que o corpo do trabalhador é insumo para produção de mercadorias e serviços. As análises de Donnangelo ${ }^{14}$ corroboram com este entendimento, ao apontar que:

Cuidar deste valoroso ente, o corpo dos trabalhadores, é uma das funções precípuas dadas ao serviço de saúde, para manutenção do sistema produtivo (...). A força de trabalho expressa através do corpo sadio é recurso fundamental à produção $e$ à acumulação de capital. Recebe determinada atenção do sistema de saúde, com objetivos vinculados á necessidade de reprodução da força de trabalho frente ao processo de produção econômica.

Outra incoerência identificada se refere aos territórios que devem ser investidos para qualificação e humanização da saúde. No manual operacional do QualiSUS-Redes (2009), uma das justificativas para a articulação das redes de serviços de saúde são as expressivas diferenças loco regionais e os vazios assistenciais em diversos territórios. No entanto, o projeto é voltado prioritariamente para regiões metropolitanas, e na Portaria 1375 de 03 de julho de 2012 que define as regiões selecionadas para participação e implementação das ações dos subprojetos do QualiSUS-Redes, o maior montante de financiamento é direcionado para as regiões metropolitanas da região sudeste e Sul do país, que são, historicamente, as regiões mais desenvolvidas do Brasil.

0 enfoque econômico e fortalecedor do modelo biomédico, pode ser visto com a prioridade do projeto às regiões com base populacional com no mínimo $250 \mathrm{mil}$ habitantes "visando garantir a maior eficiência no uso dos recursos de saúde" 15 e, a prioridade dada ao investimentos à assistência especializada de média complexidade e de assistência de urgência e emergência.

Outra questão se refere à priorização da atenção primária à saúde. Apesar de esta estar disposta como central nos documentos e ser colocada como a principal porta de entrada dos serviços de saúde, tendo a potencialidade de resolução de grande parte dos problemas de saúde quando efetiva os processos de educação, promoção e prevenção à saúde, o projeto prioriza as especialidades, principalmente os setores de urgência e emergência, e o fomento aos setores de maior complexidade.

Esta linha de intervenção é desenvolvida desde o lançamento da Política QualiSUS em 2003 e se tornou prioridade em 2004 com o projeto de Qualificação dos Serviços de Urgência nas grandes cidades ${ }^{16}$. No próprio Acordo de Empréstimo de 2009, já fica expresso a prioridade a ser dada ao 
atendimento no nível secundário, especialidades e centros de emergência e sistemas logísticos ${ }^{17}$ o que contribui diretamente para fomento da economia e do mercado, na medida em que estimula a compra e venda de produtos de alta tecnologia, maquinário e farmacêuticos para atendimento das necessidades postas pelo tratamento disposto por este nível de atenção à saúde.

Aqui se encontra um tensionamento entre a lógica de mercado e a demanda da população por serviços qualificados de urgência e emergência. A precarização de tais serviços vem sendo denunciada ${ }^{18} \mathrm{em}$ diversas instâncias e a necessidade de sua qualificação merece atenção. No entanto, neste trabalho, chama-se atenção para a lógica de fomento a economia no qual essa política se sustenta.

A partir dessa lógica, estimula-se uma ampliação do mercado privado de saúde, seja através da indústria de medicamentos e equipamentos médico-hospitalares, planos privados de saúde, seguros saúde ou através da inserção de serviços de saúde no mundo da financeirização. É nesse sentido que se corrobora com Soares ${ }^{19}$ ao destacar que o processo saúde-doença torna-se espaço de lucratividade e hegemonia política do grande capital, por meio de um movimento de precarização da política pública de saúde, da sua focalização nos segmentos mais pobres, e do ataque ao projeto de reforma sanitária construído nos anos 1980. No campo das resistências e denúncias desses processos, com uma proposta ampla de enfrentamento à privatização da saúde, tem-se a Frente Nacional que foi articulada por diversos movimentos sociais e entidades visando à construção de resistência junto às medidas regressivas e a desmercantilização das políticas sociais. A Frente Nacional contra a privatização da saúde se mobiliza na defesa da implementação do Projeto de Reforma Sanitária construído nos anos 1980 a partir do entendimento da saúde enquanto melhores condições de vida e de trabalho; e se orienta para a construção de uma sociedade justa e igualitária, apresentando cristalinamente a vinculação ao projeto de reforma sanitária socialista ${ }^{11}$.

\section{CONCLUSÃO}

Pode-se verificar que o Projeto QualiSUSRedes, se insere em um cenário direcionado por uma racionalidade que articula saúde e desenvolvimento, ou seja, coloca a saúde tanto como um direito, como uma área de investimento público e/ou privado; redefine o entendimento que é público-estatal, trazendo para o espaço público uma lógica privada de gestão, fomentada por uma racionalidade empresarial e seus instrumentos administrativo-gerenciais; e trazem o debate dos chamados "novos" modelos e instrumentos de gestão, que põe em segundo plano o interesse público, a universalidade do acesso aos serviços de saúde e o controle público das políticas sociais.

Decerto que o Programa QualiSUSRedes coloca em um contexto de desmonte das políticas sociais e de ataque aos princípios e diretrizes do SUS. O que se vê é que a justificativa para sua proposição é direcionada por uma lógica que mistifica as contradições da política de saúde e culpabiliza e responsabiliza trabalhadores e usuários pela baixa qualidade/desumanização dos serviços; ou localiza os problemas na má administração e gerência, abrindo caminho para incorporação de estratégias de gestão vinculadas ao mercado.

Desta forma, é explícita a contradição entre a tentativa de implantação de um Programa que busca democratizar as relações no atendimento dos serviços e ampliar o acesso, aumentar o diálogo entre profissionais e usuários e estabelecer estratégias de gestão compartilhada, se o cenário efetivo é de uma política de saúde com financiamento precário, baixo investimento em setores estratégicos para atendimento das necessidades dos usuários, fortalecimento do setor privado, com os trabalhadores vivenciando a precarização e a fragilização das relações de trabalho, e a população com condições de vida e trabalho cada vez mais acirradas. 
Neste sentindo, o QualiSUS-Redes acompanha as propostas do Banco Mundial para a área da saúde que trazem como elementos centrais os incentivos e o fortalecimento da prestação de contas para melhorar a eficiência e o uso dos recursos, aumentar a qualidade da assistência e conter os custos em ascensão; o fortalecimento da capacidade do sistema de saúde para lidar com a expansão das doenças não transmissíveis e a relevância das deficiências do mercado de serviços e de seguro privado.

Entende-se que o alinhamento a estas propostas fragilizam a efetivação do QualiSUS-Redes em suas potencialidades e contribuem para fortalecer uma lógica que vai de encontro ao projeto de reforma sanitária e às diretrizes e princípios do SUS e fortalecimento da lógica privatista.

\section{REFERÊNCIAS}

1. Banco Internacional para Reconstrução e Desenvolvimento. Corporação Financeira Internacional. Estratégia de parceria com o país para a República Federativa do Brasil: período de 2008 a 2011: Relatório n42677BR [Internet]. 2008 [citado em 03 fev 2014]. Disponível em: http://siteresources.worldbank.org/INTLAC BRAZILINPOR/Resources/EPP.pdf.

2. Gusmão-Filho FA, Carvalho E, Araújo-Filho JL. Avaliação do grau de implantação do Programa de Qualificação da Atenção Hospitalar de Urgência (Qualisus). Ciênc Saúde Coletiva. 2010; 15(supl 1):1227-38.

3. O'Dwyer GO, Oliveira SO, De Seta MH. Avaliação dos serviços hospitalares de emergência do programa QualiSUS. Ciênc Saúde Coletiva. 2009; 14(5):1881-90.

4. Bittencourt RJ, Hortale VA. A qualidade nos serviços de emergência de hospitais públicos e algumas considerações sobre a conjuntura recente no município do Rio de Janeiro. Ciênc Saúde Coletiva. 2007; 12(4):929-34.

5. Ministério da Saúde (Br). Plano de ações estratégicas para o enfrentamento das Doenças Crônicas Não transmissíveis (DCNT) no Brasil 2011-2022 [Internet]. Brasília (DF): Ministério da Saúde; 2011 [citado em 17 dez 2014]. Disponível em: http://bvsms.saude.gov.br/bvs/publicacoes/ plano_acoes_enfrent_dcnt_2011.pdf.

6. Conselho Nacional de Secretários de Saúde. Nota Técnica 47/2013, de 19 de abril de 2013: QualiSUS-Rede: Projeto P0088716 [Internet]. Brasília, (DF): CONASS; 2013 [citado em 03 fev 2014]. Disponível em: http://www.conass.org.br/NT\%20472013\%20-\%20QualiSUS-Rede.pdf.

7. The World Bank. Project appraisal document on a proposed loan in the amount of US $\$ 235.0$ million to the federative republic of Brazil for a health network formation and quality improvement project (QUALISUSREDE): Report no 42359-BR [Internet]. 2008 [citado em 2014 Dez 03]. Disponível em: http://documents.worldbank.org/curated/e n/2008/12/10152116/brazil-first-phaseadaptable-program-loan-health-networkformation-quality-improvement-projectbrazil-first-phase-adaptable-pr.

8. The World Bank. Project information document (PID): pprasisal stage: Report №. AB3637 [Internet]. 2008 [citado em $2014 \mathrm{Fev}$ 03]. Disponível em: http://documents.worldbank.org/curated/e n/2008/04/9362338/brazil-health-networkformation-quality-improvement-project.

9. Soares RC. A contrarreforma na política de saúde e o SUS hoje: impactos e demandas ao Serviço Social. Tese [Doutorado]. Recife: Universidade Federal de Pernambuco; 2010. 10. Governo Federal (Brasil). Brasil: 20032010 [Internet]. Brasília (DF): Governo Federal; 2010 [citado em 12 Jan 2012]. Disponível em: http://www.balancodegoverno.presidencia.g ov.br/sintese-politica/sintese-politicaversao-impressa.

11. Bravo MI, Menezes, JSB. A Saúde nos Governos Lula e Dilma: algumas reflexões. In: Bravo MI, Menezes, JSB, organizadoras. A saúde na atualidade: por um sistema único de saúde estatal, universal, gratuito e de qualidade. Rio de Janeiro: UERJ, Rede Sirus; 2011. (Cadernos de Saúde). p.15-28.

12. Pereira JMM. O Banco Mundial como ator político, intelectual e financeiro - 1944-2008. Rio de Janeiro: Civilização Brasileira; 2010.

13. Soares R. Contrarreforma na política de saúde e prática profissional do Serviço Social 
nos anos 2000. In: Mota AE, organizadora. As ideologias da contrarreforma e o Serviço Social. Recife: UFPE; 2010. p.337-382.

14. Donnangelo MCF. Medicina e Sociedade (o médico e seu mercado de trabalho). São Paulo: Pioneira; 1975.

15. Ministério da Saúde (Br). Manual operacional do projeto de investimento para a qualificação do Sistema Único de Saúde [Internet]. Brasília (DF): Ministério da Saúde; 2009 [citado em 12 jan 2012]. Disponível em: http://observasaude.fundap.sp.gov.br/RgGra ndeABC/Gesto\%20Regional/QualiSUS-

Rede\%20-

\%20regi\%C3\%A3o\%20de\%20sa\%C3\%BAde $\% 20$ Grande\%20ABC/MnlOpera_QualiSUS.pd f.

16. Gusmão-Filho FAR. Análise de implantação da Política de Qualificação da Atenção à Saúde do Sistema Único de Saúde Política QUALISUS - em três hospitais do município de Recife. Tese [Doutorado]. Recife: FIOCRUZ, Centro de Pesquisas Aggeu
Magalhães; 2008.

17. The World Bank. Loan Agreement for Loan number 7632-BR conformed [Internet]. 2009. [citado em 2014 Fev 03]. Disponível em:

http://documents.worldbank.org/curated/e n/2009/12/11710424/loan-agreementloan-7632-br-conformed.

18. Olivar $M$, Vidal D. 0 trabalho dos assistentes sociais nos hospitais de emergência: notas para o debate. Serv Soc Soc. 2007; 92:139-58.

19. Soares RA. Racionalidade da contrarreforma na política de saúde e o Serviço Social. In: Bravo MI, Menezes JSB. Saúde, Serviço Social, movimentos sociais e conselhos. São Paulo: Cortez, 2012. p. 85-108.

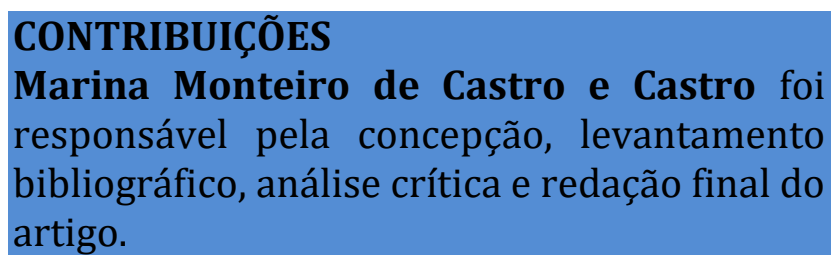

\section{Como citar este artigo (Vancouver):}

Castro MMC. Qualissus-redes e as proposições do banco mundial: problematizações críticas. REFACS [Online]. 2016 [citado em: (dia, mês, ano de acesso)]; 4(2). Disponível em: (link de acesso). DOI: $10.18554 /$ refacs.v4i2.1129.

\section{Como citar este artigo (ABNT):}

CASTRO, M. M, C. Qualissus-redes e as proposições do banco mundial: problematizações críticas. REFACS, Uberaba, MG, v. 4, n. 2, p. 153-161, 2016. DOI: 10.18554/refacs.v4i2.1129. Disponível em: (link de acesso). Acesso em: (inserir dia, mês e ano de acesso).

\section{Como citar este artigo (APA):}

Castro, M. M. C. (2016). Qualissus-redes e as proposições do banco mundial: problematizações críticas. REFACS, 4(2), 153-161. Recuperado em (dia), (mês), (ano) de (link de acesso). DOI: $10.18554 /$ refacs.v4i2.1129. 